\title{
Serum and urinary soluble $\alpha$-Klotho levels in patients with chronic kidney disease
}

\author{
Khalid J. Al-Jewari ${ }^{1}$, Rayah S. Baban ${ }^{1}$ and Jawad K. Manuti ${ }^{2}$ \\ ${ }^{1}$ Department of Chemistry and Biochemistry, College of Medicine, Al-Nahrain University, Baghdad, Iraq \\ ${ }^{2}$ Department of Internal Medicine, College of Medicine, Al-Nahrain University, Baghdad, Iraq
}

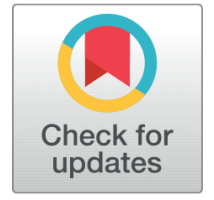

Received 10-01-2021

Revised 15-01-2021

Accepted 29-03-2021

Published 30-03-2021

\section{Corresponding Author}

Khalid J. Al-Jewari

khalid.aljawari008@gmail.com

Department of Chemistry and

Biochemistry, College of

Medicine, Al-Nahrain University,

Baghdad, Iraq

DOI https://doi.org/10.47419/ bjbabs.v2i01.35

Pages: 29-38

Distributed under the terms of the Creative

Commons

Attribution-NonCommercial 4.0 International (CC-BY-NC 4.0), which permits use for any non-commercial purpose, distribution, and reproduction in any medium, provided that the original work is properly cited.

Copyright: (C) 2021 Khalid J. Al-Jewari, Rayah S. Baban, Jawad K. Manuti

\section{ABSTRACT}

Background: Chronic kidney disease is an epidemic global health problem that leads to death. To prevent any disease progression and treatment, the diagnosis must be made in the early stage by studying renal damage. Klotho is a protein found in many organs of the human body, but it is mainly abundant in the kidney.

Objectives: This study is aimed to evaluate klotho's clinical significance as an additional biomarker for diagnosing chronic kidney disease in its early stages. Methods: One hundred subjects were included in this study to measure their serum and urinary klotho. Forty patients with chronic kidney disease (pre-dialysis) and sixty normal subjects were enrolled in this study. Serum and urinary klotho were determined using the ELISA technique in addition to other renal function tests. Results: Serum and urinary alpha klotho was decreased in CKD patients when it compared with control subjects. A positive correlation was found between serum creatinine and urinary alpha klotho in the patients' group.

Conclusion: Serum and urinary alpha klotho levels were decreased significantly in patients with chronic kidney disease compared with healthy controls.

Keywords chronic kidney disease, CDK, eGFR, soluble alpha-Klotho, urinary alpha-Klotho

\section{INTRODUCTION}

Chronic kidney disease (CKD) or chronic renal failure is a universal health problem characterized by gradual renal function loss. As renal function deteriorates, mineral metabolism disturbances, such as hyperphosphatemia, hypocalcaemia, develop obviously. These metabolic disorders are firmly connected with poor prognosis and survival. Therefore, CKD's early diagnosis and management are important to prevent progression and extrarenal complications. ${ }^{1}$ CKD is defined as abnormalities of kidney structure or function, present for $>3$ months, with health implications. The abnormalities of kidney structure or function may be recognized clinically by different criteria: just one of these criteria

\section{OPEN ACCESS}


is enough to diagnose CKD. Criteria include a decreased glomerular filtration rate (GFR) [ $<60 \mathrm{~mL} / \mathrm{min} / 1.73 \mathrm{~m}^{2}$ ) or evidence of kidney damage such as albuminuria $\geq 30 \mathrm{mg} / \mathrm{g}$, urine sediment abnormalities, electrolyte and other abnormalities due to tubular disorders. ${ }^{2} \mathrm{CKD}$ classified into five stages by according to estimated glomerular filtration rate (eGFR). ${ }^{3}$ as showed in table-1.

\begin{tabular}{lll}
\hline \multicolumn{2}{l}{ Table 1} & Stages of chronic kidney disease \\
\hline Stage & Qualitative Description & GFR $\left(\mathbf{m L} / \mathbf{m i n} . / \mathbf{1 . 7 3 m ^ { 2 }}\right)$ \\
1 & Kidney damage-Normal GFR & $>90^{*}$ \\
2 & Kidney damage- Mild decreased GFR & $60-89^{*}$ \\
$3 \mathrm{a}$ & Moderate decreased GFR & $45-59$ \\
$3 \mathrm{~b}$ & Moderate decreased GFR & $30-44$ \\
4 & Severe decreased GFR & $15-29$ \\
5 & End-stage renal disease & $<15$ \\
\hline
\end{tabular}

${ }^{\star} A G F R>60 \mathrm{~mL} / \mathrm{min} . / 1.73 \mathrm{~m}^{2}$ in isolation is not CKD unless other kidney damage evidence is present.

Klotho is a transmembrane $130 \mathrm{KDa}$ protein. ${ }^{4}$ It is considered an anti-ageing protein predominantly produced in the kidney and several other tissues, including the parathyroid glands and the choroid plexus's epithelial cells. There are two forms of $\alpha$-klotho, including membrane $\alpha$-klotho and secreted $\alpha$-klotho. ${ }^{5}$ In the kidney, Klotho serves as a co-receptor for FGF23 to down-regulate the sodium-phosphate transporters NaPi2a and NaPi2c and promote renal phosphate excretion. Dysfunction in the Klotho/FGF23 axis and associated disordered phosphate metabolism have been suggested as a potential pathological mechanism by which Klotho deficiency may drive CKD progression. ${ }^{6}$

Klotho deficiency is involved in acute and chronic kidney diseases, cancers, and saltsensitive hypertension. The serum level of Klotho decreases with ageing in humans. However, the biological function of Klotho and how Klotho deficiency contributes to age-related diseases remain elusive. ${ }^{7}$

Further studies disclosed that acute kidney injury (AKI) and chronic kidney disease (CKD) are conditions of acquired Klotho deficiency. Indeed, several of the features of genetic Klotho deficiency, such as bone disease, vascular calcification, left ventricular hypertrophy, premature mortality, increased FGF23 levels and hyperphosphatemia, among others, are also features of CKD. ${ }^{8}$

Chronic kidney disease (CKD) is increasingly considered a major public health issue worldwide with high mortality and morbidity rates. Currently, there is no effective therapy available for treating $\mathrm{CKD}$. Therefore, early detection or prognosis is important for the prevention and treatment of CKD. However, there is no standard biomarker for early diagnosis and the monitoring of disease exacerbation in the course of CKD. Emerging evidence from patients with CKD has shown that s. Klotho levels are decreased in the early stages of $\mathrm{CKD}$, and they further decline as CKD progresses. Moreover, reduced s. Klotho levels are associated with an elevated risk of deterioration in renal function or renal replacement treatment (RRT). Therefore, s. Klotho is proposed as a biomarker for the early diagnosis and progression of CKD. ${ }^{9}$ 


\section{MATERIALS AND METHODS Study design, subjects and variables}

A case-control study was conducted from December 2019 to March 2020 at the Chemistry and Biochemistry Department, College of Medicine, Al-Nahrain University, and samples taken from patients referred to the Department of General Medicine/Urology at AlImamain Al- khadhimain Medical City in Iraq- Baghdad. They were divided into two groups: case group, which includes forty patients who suffer from chronic kidney disease in the first, second and third stages. That was selected depends on inclusion criteria and a control group that includes sixty healthy subjects.

Patients with renal impairment and age $>18$ years, diabetic nephropathy, chronic glomerulonephritis, nephritis, obstructive nephropathy, hypertensive nephropathy were included in the study. Patient with renal transplantation, patient with comorbidity of other diseases such as chronic obstructive airway disease were excluded from the study.

The Institutional Review Board (IRB) of Al-Nahrain University, College of Medicine approved the research protocol, and all subjects included in the present study provided their written informed consent.

Patients diagnosed with CKD in early-stages (stages 1, 2, and 3) only were selected. Using the MDRD equation, all subjects were selected must be $>18$ years and within inclusion criteria.

Five $\mathrm{ml}$ of blood were taken from both patients and control subjects using a gel tube, centrifuge it at $4000 \mathrm{rpm}$ for 10 minutes to obtain serum for serum creatinine, Urea, Uric Acid Po4, $\mathrm{Ca}^{++}{ }_{\text {,eGFR }}$ and serum soluble alpha klotho. Also, $5 \mathrm{ml}$ of fresh urine in the morning was taken from each patient and controls to measure urine alpha klotho and Microalbuminuria. The human soluble alpha klotho level was measured using the ELISA kit (Sunlong biotech co., LTD) for quantitative determination concentration in serum and urine samples.

\section{Statistical analysis}

This case-control study's statistical analysis was performed with (SPSS) version-24, and Microsoft Excel operation formulated categorical data as count and percentage. Tester with chi-square. Numerical data were described as mean and standard deviation, analysis of variance ( $t$-test) used to compare two groups and the least difference option used to identify the difference.

\section{RESULTS}

CKD patients group showed significantly lower serum $\alpha$ - klotho $(489.93 \pm 91.64)$ and urinary $\alpha$ - klotho $(369.15 \pm 143.46)$ as compared with control group $(531.08 \pm 106.76, \mathrm{p}=$ $0.04)$ and $(449.06 \pm 118.41, \mathrm{P}=0.005)$ respectively. 
Descriptive statistics was conducted for eGFR, S.PO4, Microalbuminuria, $\mathrm{Ca}^{+2}$, Uric acid, serum creatinine and Urea for both groups in the study. For CKD, the eGFR had an average of $45.19 \mathrm{ml} / \mathrm{min} \pm 10.26$, which was significantly lower than controls, which had an average of $(121.95 \pm 23.17, \mathrm{p}<.001)$. For CKD, the serum PO4 had an average of $6.78 \pm$ 2.01 significantly higher than controls, $(3.59 \pm 0.75, \mathrm{p}<.001)$, Micro- albuminuria had an average of $114.80 \pm 69.08$ for CKD, which is higher than that of controls $(12.27 \pm 5.16$, $p$ $<.001)$. Serum $\mathrm{Ca}^{+2}$ had an average of $(7.15 \pm 0.86, \mathrm{p}<0.001)$ in CKD patients, which is significantly lower than that of controls, $(9.12 \pm 0.66, \mathrm{p}<0.001)$. Also, the observations of serum uric acid in CKD had an average of 8.39 ( $\mathrm{SD}=1.23$, $\mathrm{SEM}=0.19$, Min $=5.50$, $\mathrm{Max}=11.20)$ which is significantly higher than controls $4.42(\mathrm{SD}=1.30, \mathrm{SEM}=0.17$, Min $=2.00, \mathrm{Max}=7.40, \mathrm{p}<0.001)$. For CKD, the $\mathrm{S}$. Creatinine had an average of $1.54 \pm 0.27$ significantly higher than that for controls $(0.71 \pm 0.12, \mathrm{p}<0.001)$. Finally, serum Urea had also significant higher value $96.39 \pm 39.25$, in CKD patients than in controls $(27.50 \pm 6.27$, $\mathrm{p}<0.001)($ table-2).

\begin{tabular}{|c|c|c|c|c|c|c|}
\hline Variable & Cases & Mean \pm SD & $\mathrm{SE}_{\mathrm{M}}$ & Min. & Max. & $\begin{array}{l}P \\
\text {-value }^{*}\end{array}$ \\
\hline \multirow{2}{*}{$\begin{array}{l}\text { eGFR } \\
\left(\mathrm{mL} / \mathrm{min} . / 1.73 \mathrm{~m}^{2}\right)\end{array}$} & CKD & $45.19 \pm 10.26$ & 1.62 & 23 & 64.79 & $<0.001$ \\
\hline & Controls & $121.95 \pm 23.17$ & 2.99 & 91 & 186.0 & \\
\hline \multirow[t]{2}{*}{ S. PO4 (mg/dl) } & CKD & $6.78 \pm 2.01$ & 0.32 & 2.1 & 12 & $<0.001$ \\
\hline & Controls & $3.59 \pm 0.75$ & 0.10 & 2.1 & 4.7 & \\
\hline \multirow[t]{2}{*}{ Urinary albumin (g/dl) } & CKD & $114.80 \pm 69.08$ & 10.92 & 22.000 & 374.3 & $<0.001$ \\
\hline & Controls & $12.27 \pm 5.16$ & 0.67 & 2.500 & 20.0 & \\
\hline \multirow[t]{2}{*}{ S. $\alpha$-klotho(pg/ml) } & CKD & $489.93 \pm 91.64$ & 14.49 & 311.875 & 683.77 & 0.042 \\
\hline & Controls & $531.08 \pm 106.76$ & 13.78 & 261.286 & 764.45 & \\
\hline \multirow[t]{2}{*}{ U. $\alpha$-Klotho(pg/ml) } & CKD & $369.15 \pm 143.46$ & 22.68 & 33.345 & 748.51 & 0.005 \\
\hline & Controls & $449.06 \pm 118.41$ & 15.29 & 169.51 & 854.842 & \\
\hline \multirow[t]{2}{*}{$\mathrm{Ca}^{+2}(\mathrm{mg} / \mathrm{dl})$} & CKD & $7.15 \pm 0.86$ & 0.14 & 5.4 & 8.80 & $<0.001$ \\
\hline & Controls & $9.12 \pm 0.66$ & 0.09 & 7.5 & 10.10 & \\
\hline \multirow[t]{2}{*}{ S. Cr. ( mg/dl) } & CKD & $1.54 \pm 0.27$ & 0.04 & 1.17 & 2.30 & $<0.001$ \\
\hline & Controls & $0.71 \pm 0.12$ & 0.02 & 0.6 & 0.9 & \\
\hline \multirow[t]{2}{*}{ Urea (mg/dl) } & CKD & $96.39 \pm 39.25$ & 6.21 & 40.0 & 200.0 & $<0.001$ \\
\hline & Controls & $27.50 \pm 6.27$ & 0.81 & 19.0 & 41.0 & \\
\hline \multirow[t]{2}{*}{ Uric acid (mg/dl) } & CKD & $8.39 \pm 1.23$ & 0.19 & 5.50 & 11.20 & $<0.001$ \\
\hline & Controls & $4.42 \pm 1.30$ & 0.17 & 2.00 & 7.40 & \\
\hline
\end{tabular}

- ${ }^{P}$ values for testing the mean differences by subjects using t-test.

- The number of participants in the CKD group is 40 and in the controls is 60 .

Regarding correlation, a significant negative correlation was observed between Age and eGFR $\left(\mathrm{r}_{p}=-0.41, \mathrm{p}=.010,95 \%\right.$ CI $\left.[-0.64,-0.11]\right)$. The correlation coefficient between Age and eGFR was -0.41 , which is considered a moderate effect size. A significant positive correlation was observed between Age and UA $\left(\mathrm{r}_{p}=0.35, \mathrm{p}=0.027,95 \%\right.$ CI $\left.[0.04,0.60]\right)$. eGFR significantly and negatively correlated with both UA and Micro Alb $\left(\mathrm{r}_{p}=-0.42, \mathrm{p}=\right.$ 
$.008,95 \% \mathrm{CI}[-0.65,-0.12])$ and $\left(\mathrm{r}_{p}=-0.32, \mathrm{p}=.044,95 \% \mathrm{CI}[-0.58,-0.01]\right)$ respectively The correlation between eGFR and Micro Alb, are of moderate effect size. A significant negative correlation was observed between duration and eGFR $\left(\mathrm{r}_{p}=-0.43, \mathrm{p}=.007,95 \%\right.$ CI $[-0.65$, -0.13]). The correlation coefficient between duration and eGFR was -0.43 . A significant negative correlation was observed between S. Cr and eGFR $\left(\mathrm{r}_{p}=-0.57, \mathrm{p}<.001,95 \%\right.$ CI $[-0.75,-0.31])$. The correlation coefficient between S. Cr and eGFR was -0.57 , indicating a large effect size. A significant positive correlation was observed between S. Cr and Urinary klotho $\left(\mathrm{r}_{p}=0.35, \mathrm{p}=.029,95 \%\right.$ CI $\left.[0.04,0.60]\right)$. The correlation coefficient between S. Cr and Urinary klotho was 0.35 , indicating a moderate effect size. This correlation indicates that as S. Cr increases, Urinary klotho tends to increase as shown in Table 3.

\begin{tabular}{llll}
\hline \multicolumn{2}{l}{ Table 3 Significant Pearson correlations among different parameters in the study groups } \\
Combination & $\mathbf{r}_{p}$ & $\mathbf{9 5 \%}$ CI & $\boldsymbol{p}$ \\
Age- eGFR & -0.41 & {$[-0.64,-0.11]$} & $\mathbf{. 0 1 0}$ \\
Age- UA & 0.35 & {$[0.04,0.60]$} & $\mathbf{. 0 2 7}$ \\
duration- eGFR & -0.43 & {$[-0.65,-0.13]$} & $\mathbf{. 0 0 7}$ \\
B. Urea-uric acid & 0.37 & {$[0.06,0.61]$} & $\mathbf{. 0 2 1}$ \\
S. Cr-eGFR & -0.57 & {$[-0.75,-0.31]$} & $<.001$ \\
S. Cr- U. $\alpha$-klotho & 0.35 & {$[0.04,0.60]$} & $\mathbf{. 0 2 9}$ \\
eGFR-UA & -0.42 & {$[-0.65,-0.12]$} & $\mathbf{. 0 0 8}$ \\
eGFR- Microalbuminuria & -0.32 & {$[-0.58,-0.01]$} & $\mathbf{. 0 4 4}$ \\
UA-S_PO4 & 0.40 & {$[0.10,0.64]$} & $\mathbf{. 0 1 1}$ \\
Ca-S_PO4 & -0.41 & {$[-0.64,-0.11]$} & $\mathbf{. 0 1 0}$ \\
Age- eGFR & -0.51 & {$[-0.68,-0.30]$} & $<.001$ \\
Age- Ca ${ }^{+2}$ & 0.29 & {$[0.04,0.51]$} & $\mathbf{. 0 2 4}$ \\
S. Cr- eGFR & -0.40 & {$[-0.60,-0.16]$} & $\mathbf{. 0 0 1}$ \\
UA-Ca & -0.25 & {$[-0.48,0.00]$} & .051 \\
Ca-U. klotho & -0.23 & {$[-0.46,0.02]$} & .074 \\
\hline
\end{tabular}

Also, ROC analysis was done for eGFR and serum and urinary $\alpha$ - Klotho. Estimated GFR had AUC = 1, indicating an excellent parameter for discrimination between CKD and controls; this study had $100 \%$ sensitivity and specificity at threshold value $=64.79$.

For serum $\alpha$ - Klotho, it had an AUC value of 0.627 with the sensitivity of $65 \%$ and specificity of $66.7 \%$ at a cut off value of 509.28 , indicating that serum $\alpha$-Klotho is poor in distinguishing between $\mathrm{CKD}$ and normal subjects. However, it showed a significantly low value in CKD patients compared with controls. For urinary $\alpha$ - Klotho, the AUC value was 0.683 with a sensitivity of $80 \%$ and specificity of $53.3 \%$ at the cut off value of 449.58 , indicating that urine $\alpha$-Klotho also has a poor distinguishing ability between CKD and normal subjects, as shown in figure- 1 and 2. 


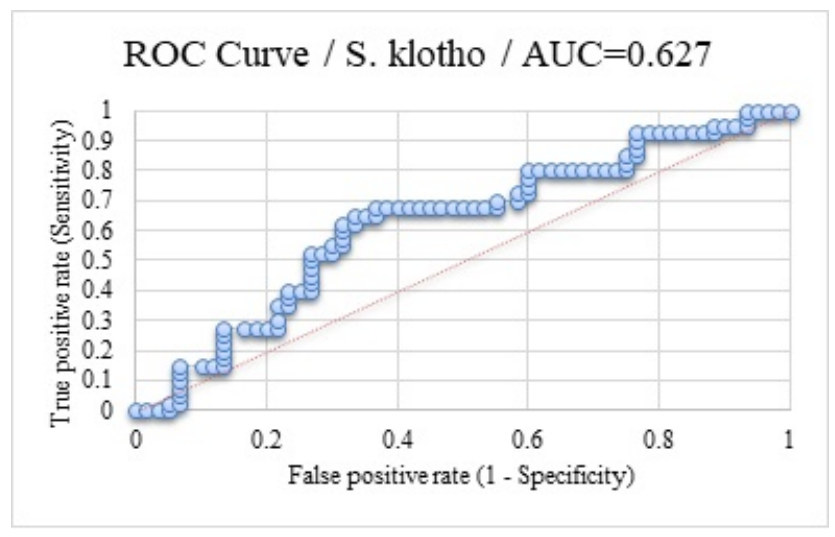

Figure 1 ROC Curve for S.klotho in patients with CKD as compared with controls. values less than the threshold is considered positive for the disease

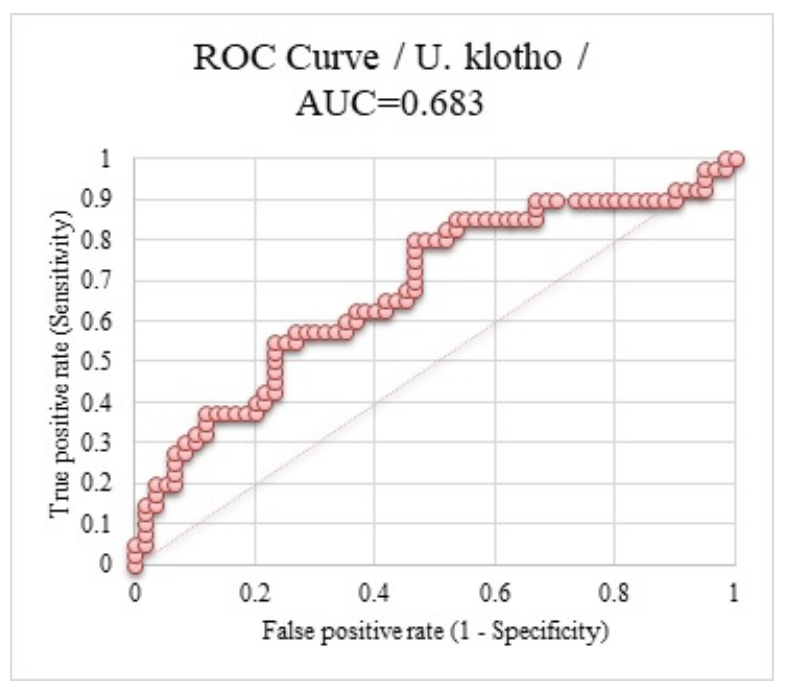

Figure 2 ROC Curve for U. klotho in patients with CKD as compared with controls. values less than the threshold is considered positive for the disease 


\section{DISCUSSION}

The decrease in serum and urinary soluble alpha klotho level of patients group with CKD (stages 1, 2 and 3) compared with the control group (table-2) can be discussed as follow; as renal is the major source of klotho, any simple damage occurs in it will affect its level, and so the klotho level will be decreased. This decrease may found in various conditions associate with renal dysfunction, such as hypertension or during ageing (Picciotto et. al., 2019). ${ }^{4}$ Our results were agreed with those of Neyra et. al. (2016), ${ }^{10}$ who reported that the circulation of soluble alpha klotho start to decline in stage 1,2 in the urine of a patient with chronic kidney disease. Also, Lu et. al. (2017) ${ }^{11}$ have demonstrated a decline in serum and urinary Klotho, followed by a rise in serum FGF23 at an early stage of chronic kidney disease (CKD).

Regarding correlation, the statistical result showed a significant positive correlation between urinary soluble alpha klotho and serum creatinine, table 3 . This result has disagreed with those Seo et. al. (2015), ${ }^{12}$ who found a negative correlation between urinary soluble alpha klotho and serum creatinine level. They have suggested that renal soluble alpha Klotho expression decreases significantly at some point after the damage. The disagreement is due mostly to our small sample size. In contrast, Lee et. al. (2014) ${ }^{13}$ results support our findings indirectly because this study includes diabetic data; they show, for the first time, high urine $\alpha$-klotho levels, which is found to be associated with diabetes in humans even in the normoalbuminuria stage, and report a high urine $\alpha$-klotho levels were observed to be associated with diabetes in humans even in the normoalbuminuria stage, and express the reason of present high klotho level in urine due to filtration by glomeruli and attribute the reason to hyperglycemia does not affect renal $\alpha$-klotho production. Because the increased glucose level does not alter $\alpha$-klotho expression in kidney cells, and diabetes does not affect renal $\alpha$-klotho mRNA expression.

ROC analysis for serum $\alpha$ - Klotho had an AUC value of 0.627 with a sensitivity of $65 \%$ and a specificity of $66.7 \%$ at a cut off value of 509.28 , indicating that serum $\alpha$-Klotho is poor distinguishing between $\mathrm{CKD}$ and normal subjects. However, it showed a significantly low value in CKD patients compared with controls. For urinary $\alpha$ - Klotho, the AUC value was 0.683 with a sensitivity of $80 \%$ and specificity of $53.3 \%$ at cut off value of 449.58 , indicating that urine $\alpha$-Klotho also has poor distinguishing ability between CKD and normal subjects. Khodeir et. al. (2019) ${ }^{1}$ showed high specificity $100 \%$ and sensitivity of $92 \%$ and cutoff value $<9.9$ to decrement between normal and CKD patient.

In this study, eGFR has a highly significant decrease ( $\mathrm{p}$-value $<0.001$ ) in patients with chronic kidney disease as compared with control respectively $(45.19 \pm 10.26)$ vs $(121.95$ \pm 23.17 ) as shown in table-2, this result explain the eGFR decline with the progression stages of chronic kidney disease, which agreed with Tsai et. al. (2017) findings; ${ }^{14}$ they reported that demonstrated eGFR decline rate performed well to predict chronic kidney disease progression and end-stage of the renal disease events. The predictive performance may be better than the baseline chronic kidney disease stages.

A significant negative correlation was found between age and eGFR due to increased ageing, where the number of nephrons will be gradually lost. So, eGFR will slightly be 
decreased with getting aged. Table-3 agree with the findings of Melsom et. al. (2019). ${ }^{15}$

Concerning eGFR, a highly significant inverse relation was found between eGFR and serum creatinine. The significant relation is due to renal impairment. When it's found, this impairment will lead to increased creatinine and decline eGFR according to the MDRD equation. ${ }^{16}$ Also, they have reported the same findings, negative correlation between s.creatinine and eGFR as CKD impaired renal function. Thus, the fraction of creatinine cleared by tubular secretion increased with progressive depression of GFR

\section{CONCLUSIONS}

There is a positive correlation between urinary soluble alpha klotho and serum creatinine. Soluble alpha klotho is decreased in serum and urine of patients with chronic kidney disease significantly. But according to the ROC test, and because of the small sample size, soluble klotho was considered a poor biomarker to discriminate between CKD subjects and normal subjects.

\section{ACKNOWLEDGMENTS}

None.

\section{DECLARATIONS}

\section{Authors' contributions}

All authors have equally contributed to this work. They were all reviewed and approved this paper before publication.

\section{Conflict of interest}

The authors declare that there is no conflict of interest.

\section{Ethical approvals}

This research was approved by the Institutional Review Board (IRB) at the College of Medicine, Al-Nahrain University; and before participation, each participant was given an idea about the study and her written informed consent was taken.

\section{Data availability}

The data associated with this work can be requested from the corresponding author.

\section{Funding resources}

This work was't receive any fund. 


\section{REFERENCES}

1. Khodeir S, Okda H, Abdalal HM. Clinical significance of fibroblast growth factor-23 and soluble alpha klotho in different stages of chronic kidney disease. Saudi Journal of Kidney Diseases and Transplantation. 2019;30(1):108-118. Available from: 10. 4103/1319-2442.252900;https://dx.doi.org/10.4103/1319-2442.252900.

2. Perez-Gomez MV, Bartsch LA, Castillo-Rodriguez E, Fernandez-Prado R, Fernandez-Fernandez B, Martin-Cleary C, et al. Clarifying the concept of chronic kidney disease for non-nephrologists. Clinical Kidney Journal. 2019;12(2):258-261. Available from: 10.1093/ckj/sfz007; https://dx.doi.org/10.1093/ckj/sfz007.

3. Garland JS. Elevated body mass index as a risk factor for chronic kidney disease: current perspectives. Diabetes, Metabolic Syndrome and Obesity: Targets and Therapy. 2014;7:347-355.

4. Picciotto D, Murugavel A, Ansaldo F, Rosa GM, Sofia A, Milanesi S, et al. The Organ Handling of Soluble Klotho in Humans. Kidney and Blood Pressure Research. 2019;44(4):715-726. Available from: 10.1159/000501316;https://dx.doi. org/10.1159/000501316.

5. 6-Liu X, Niu Y, Zhang X, Zhang Y, Yu Y, Huang J, et al. Recombinant $\alpha$-Klotho Protein Alleviated Acute Cardiorenal Injury in a Mouse Model of LipopolysaccharideInduced Septic Cardiorenal Syndrome Type 5. Analytical Cellular Pathology. 2019;2019. Available from: 10.1155/2019/5853426.

6. Zhong X, Jagarlapudi S, Weng Y, Ly M, Rouse JC, McClure K, et al. Structurefunction relationships of the soluble form of the anti-aging protein Klotho have therapeutic implications for managing kidney disease. Journal of Biological Chemistry. 2020;295(10):3115-3133. Available from: 10.1074/jbc.RA119.012144.

7. Kim JH, Hwang KH, Park KS, Kong ID, Cha SK. Biological role of anti-aging protein Klotho. Journal of Lifestyle Medicine . 2015;5(1):1-6. Available from: 10.15280/jlm. 2015.5.1.1.

8. Sanchez-Niño MD, Fernandez-Fernandez B, Ortiz A. Klotho, the elusive kidneyderived anti-ageing factor . Clinical Kidney Journal. 2020;13(2):125-127. Available from: https://doi.org/10.1093/ckj/sfz125.

9. Liu QF, Yu LX, Feng JH, Sun Q, Li SS, Ye JM. The Prognostic Role of Klotho in Patients with Chronic Kidney Disease: A Systematic Review and Meta-analysis. Disease markers. 2019;2019(Article ID 6468729):1-12. Available from: https://doi.org/ 10.1155/2019/6468729.

10. Neyra JA, Hu MC. $\alpha$ Klotho and chronic kidney disease. In: Litwack G, et al., editors. Vitamins \& Hormones . vol. 101. Academic Press; 2016. p. 257-310. Available from: https://doi.org/10.1016/bs.vh.2016.02.007.

11. Lu X, Hu MC. Klotho/FGF23 axis in chronic kidney disease and cardiovascular disease. Kidney Diseases. 2017;3(1):15-23. Available from: https://doi.org/10.1159/ 000452880.

12. Seo MY, Yang J, Lee JY, Kim K, Kim SC, Chang H, et al. Renal Klotho expression in patients with acute kidney injury is associated with the severity of the injury. The 
Korean Journal of Internal Medicine. 2015;30(4):489. Available from: 10.3904/kjim. 2015.30.4.489.

13. Lee EY, Kim SS, Lee JS, Kim IJ, Song SH, Cha SK, et al. Soluble $\alpha$-klotho as a novel biomarker in the early stage of nephropathy in patients with type 2 diabetes. PLoS One. 2014;9(8):e102984. Available from: 10.1371/journal.pone.0102984.

14. Tsai CW, Ting IW, Yeh HC, Kuo CC. Longitudinal change in estimated GFR among CKD patients: A 10-year follow-up study of an integrated kidney disease care program in Taiwan. PLoS One. 2017;12(4):e0173843. Available from: 10.1371/journal. pone.0173843.

15. Melsom T, Nair V, Schei J, Mariani L, Stefansson VTN, Harder JL, et al. Correlation Between Baseline GFR and Subsequent Change in GFR in Norwegian Adults Without Diabetes and in Pima Indians. American Journal of Kidney Diseases. 2019;73(6):777-785. Available from: 10.1053/j.ajkd.2018.11.011;https://dx.doi.org/ 10.1053/j.ajkd.2018.11.011.

16. Kumaresan R, Giri P. A Comparison of Serum Cystatin C and Creatinine with Glomerular FiltrationRate in Indian Patients with Chronic Kidney Disease. Oman Medical Journal. 2011;26(6):421-425. Available from: 10.5001/omj.2011.107;https: //dx.doi.org/10.5001/omj.2011.107. 